\title{
Radio Resource Management in OFDMA-based Cellular Networks Enhanced with Fixed and Nomadic Relays
}

\author{
Mohamed Salem ${ }^{1}$, Abdulkareem Adinoyi ${ }^{1}$, Halim Yanikomeroglu ${ }^{1}$, and Young-Doo Kim ${ }^{2}$ \\ ${ }^{1}$ Department of Systems and Computer Engineering, \\ Carleton University, Ottawa, Canada \\ ${ }^{2}$ Samsung Electronics, SAIT, Korea
}

\begin{abstract}
The provision of very high data rates in a ubiquitous manner throughout the service area is a great challenge for 4G and beyond-4G wireless networks. Towards that end, the deployment of fixed relays by the operators has become an accepted radio access network concept in various standardization activities including LTE-A and 802.16j. It is envisaged that next-generation networks will comprise a plethora of wireless relay stations. Worthy of mention is the plug-and-play type of relay known as nomadic relay. We devise novel radio resource management (RRM) schemes to facilitate the operation of fixed relay stations (FRSs) and nomadic relay stations (NRSs) in OFDMA-based multicellular networks. Two schemes of different decentralization levels are devised and classified as distributed and semi-centralized. A novel user-based dynamic routing or link selection that significantly reduces the feedback overhead is employed. We develop methods by which the NRSs act autonomously to acquire radio resources without relying on a central entity. NRS operation is the same in the two schemes and can be extended to any other OFDMA-based RRM scheme. Through the asynchronous opportunistic medium access of the NRSs, smart and opportunistic intra-cell channel reuse is attained. This is different from the static intra-cell reuse patterns often adopted in literature. Furthermore, we introduce a method to enable the cooperation between an NRS and a serving FRS to assist a troubled wireless terminal (WT). We thus establish the concept of nomadic relay-augmented fixed relay networks. To the extent of our knowledge of the literature, no other work has undertaken this task so far.
\end{abstract}

Index Terms - OFDMA, RRM, multihop, relaying, nomadic, intra-cell reuse, dynamic routing, scheduling, cooperation.

\section{INTRODUCTION}

This paper describes decentralized radio resource management methods devised for OFDMA-based multicellular networks enhanced with a mix of fixed relay stations (FRSs) and nomadic relay stations (NRSs). The licensed frequency band is assumed. Since bandwidth is scarce and expensive, the need for bandwidth-efficient resource management schemes can not be overemphasized. Moreover, prospective wireless services are bandwidth intensive and expected to be at a quality comparable to that provided by the wireline. However, wireless channels are quite different from those of the wirelines. The problems of co-channel interference (CCI), multi-path fading and shadowing constitute a tremendous challenge in wireless networks. To that end, researchers in both academia and industry have recognized that Orthogonal Frequency Division

A patent filing from this work has been made by Samsung Electronics Co. Ltd., SAIT, Korea. Korean patent application no: P2009-0084026. Email: $\{$ mrashad, adinoyi, halim\}@sce.carleton.ca, yd76.kim@samsung.com.
Multiple Access (OFDMA) and relaying techniques represent the most viable technology to address the challenges and meet those requirements for future wireless services. It is envisaged that a plethora of relay stations will form an integral part of the next-generation network architecture. Worthy of mention is the plug-and-play type of relay known as nomadic relay, an idea that has been entrenched in the IEEE 802.16 standards [1], [2], and gaining widespread acceptance. While mobile relay stations are characterized by their mobility (e.g., rooftop-mounted vehicular devices), NRSs are technically stationary devices but portable.

In the survey paper [3], motivational scenarios for using mobile multihop relaying are provided. Therein, NRSs can be deployed to provide a temporary coverage and capacity in an area where FRSs may not provide the required QoS. Example of temporary coverage areas could be, in general, where wireless connectivity is required for only a short period of time, such as in trade fairs and sporting events or in disaster recovery situations. In addition, NRSs can be used to spread the capacity in a large building. In such cases, NRSs will coexist with FRSs yet with potentially much better communication links to the wireless terminals (WTs).

To the extent of our knowledge, no work so far has provided mechanisms for integrating the autonomous NRSs into the cellular network or suggested the underlying RRM schemes and protocols to facilitate their coexistence with FRSs. Since the current literature does not cover the systems and architecture considered in this paper, we will refer to relevant works in the literature of decentralized RRM in OFDMA-based networks within our discussions.

In this work, we investigate the downlink (DL) operation of a visionary wireless network model, where FRSs are augmented by NRSs with the aim of providing a more reliable cost-effective service. These NRSs will be often acting as intermediate nodes between a serving FRS and a WT forming a three-hop communication. To alleviate the burden on system resources due to multihop relaying, intelligent RRM schemes are needed to facilitate aggressive resource reuse and meanwhile combat the potential CCI through opportunistic reuse and interference avoidance mechanisms. The research contributions in this paper can be summarized as follows:

- We develop novel limited-feedback decentralized RRM schemes to facilitate the operation of FRSs, the integration of NRSs, and CCI mitigation in OFDMA-based multicellular networks. 


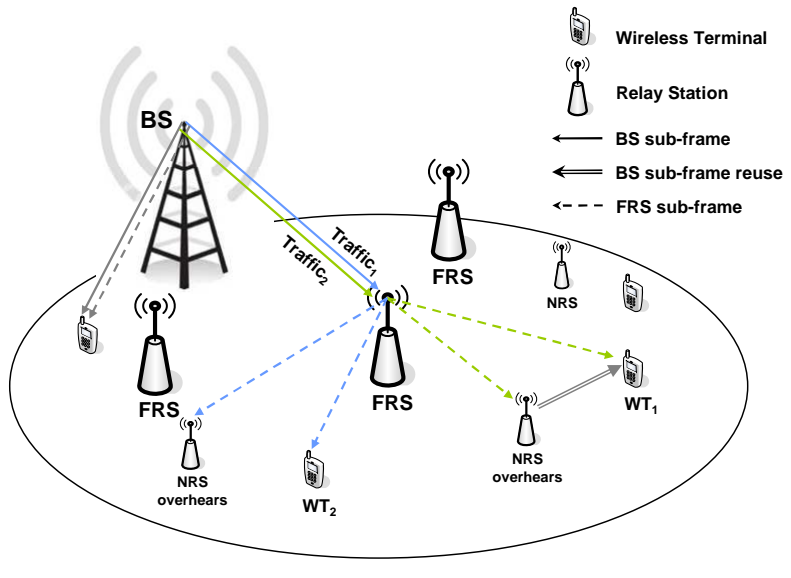

Fig. 1. The region of interest may represent a cell of the hexagonal grid, a sector of a cell, or a 'cell' of the LTE-A eNB, in the multicellular network. All resources are available in this region.

- We devise a dynamic user-based routing strategy that significantly reduces the amount of feedback overhead and achieves interference avoidance.

- A medium access technique by which NRSs autonomously acquire radio resources is introduced. NRSs asynchronously sense the activities of the subchannels around the WT that needs assistance and autonomously acquire the subchannels that have least activity. This can succinctly be described as: Listen, Acquire Resources, then Assist (LARA). This technique can be imported into any other RRM scheme.

- Through the asynchronous opportunistic medium access of NRSs, a smart intra-cell reuse that exploits the channel and interference conditions is achieved in contrast to the commonly adopted static reuese patterns.

- We devise a protocol to enable the cooperation between an NRS and an FRS to assist a troubled WT. The cooperation is based on selective relaying, meaning that the NRS only helps its WT on request and it cooperates only on the data segments it received reliably.

- Finally, we conduct extensive performance evaluation of the proposed schemes and techniques under realistic channel models and system parameters.

- Thus, we establish the concept of nomadic relayaugmented fixed relay networks.

The rest of this paper expounds on the above bullets.

\section{Descriptions of the Proposed Systems}

A representative partial network of the proposed system is shown in Fig. 1. It consists of a base station (BS), FRSs, WTs, and NRSs. The figure demonstrates the DL transmissions over two sub-frames and the half-duplex operation of all relay stations. The system shown operates in time-division duplexing (TDD) mode but can be easily applied in frequency-division duplexing (FDD). The basic allocation unit is the OFDM subchannel comprising a number of adjacent subcarriers; there are $N$ data subchannels for each the channel fading is flat. All resources are available in each region similar to that shown in Fig. 1. Adaptive modulation (AM) is employed as in [4] but only at BSs and FRSs; the achievable rate on subchannel $n$ of the link from source $m$ to destination $k$, at a particular target bit error rate $P_{e}$ and subchannel bandwidth $W$, is an upper-bounded function of the received signal-to-interferenceplus-noise ratio (SINR) $\gamma_{m, k, n}$, considering the CCI observed in the previous DL frame as in (1).

$$
R_{m, k, n}=W \min \left\{\log _{2}\left(1+\frac{-1.5 \gamma_{m, k, n}}{\ln \left(5 P_{e}\right)}\right), 10\right\} \text { bps. }
$$

The DL frame is partitioned into two consecutive equallength sub-frames. An FRS receives only during the first sub-frame (BS sub-frame) and transmits during the second (FRS sub-frame) as shown in Fig. 2. Note that the set of subchannels dynamically assigned to the feeder link of $\mathrm{FRS}_{m}$ is allocated among its connected WTs for the second hop of the same DL frame. As such, transmissions of different FRSs in the same region are maintained orthogonal during the FRS sub-frame. Such approach offers a compromise between the multiuser/frequency diversity gains and the CCI often realized in distributed schemes due to collisions of the uncoordinated allocation decisions of FRSs. A similar yet more stringent constraint has been imposed in [5] where the user data must be scheduled on the same subcarrier over the two hops.

There are $M$ FRSs deployed in the considered region at strategic locations where good line-of-sight (LOS) communication is maintained and highly directional antennas are employed. As such, BS-FRS feeder links can be assumed more immune to CCI and to experience less shadowing and smallscale fading as compared to user access links. We therefore assume that the achievable rates on the feeder link of $\mathrm{FRS}_{m}$ are sufficiently good compared to those on its users' access links so that for any set of subchannels $\mathcal{N}_{m \rightarrow k} \subseteq \mathcal{N}_{m}$ assigned for the access of relayed user $k$ during the FRS sub-frame, there exists another set $\mathcal{N}_{0 \rightarrow m, k} \subseteq \mathcal{N}_{m}$ that can be used on the feeder link such that:

$$
\sum_{j \in \mathcal{N}_{0 \rightarrow m, k}} R_{0, m, j} \geq \sum_{i \in \mathcal{N}_{m \rightarrow k}} R_{m, k, i}, \quad \forall m \neq 0
$$

where $\mathcal{N}_{m}$ is the set of subchannels assigned by the BS (node 0) to $\mathrm{FRS}_{m}$ and $\bigcap_{m=0}^{M} \mathcal{N}_{m}=\phi$. Therefore, the end-toend capacity using equal sub-frames is governed only by the relayed user's achievable rates on the second hop; this is inline as well with the decentralized nature of the system where a WT may not know the channel states of each potential feeder link. The deployment of FRSs could be different from a region to another since our schemes are not attached to a certain geography. The WT dynamically selects one access link out of $M+1$ rather than the static relay selection commonly adopted in literature, e.g., [5], and [6].

There are $K$ active WTs in the considered region and $K_{n o m}$ NRSs. Depending on the scenario of interest, the number of NRSs may vary with respect to $K$. For the sake of illustration, we have considered the case in which $K_{\text {nom }}=K$; that reflects on a scenario where each WT has placed, or selected out of many, an NRS that is dedicated to assist it on demand. The NRS is thus stationary, in a close vicinity of the WT, and often has a very good connection to the WT; likely an LOS. The NRS could be larger than mobile-phone kind of terminals and thus can employ more antennas to strengthen its link to either the serving FRS or the WT through beam steering. Note 


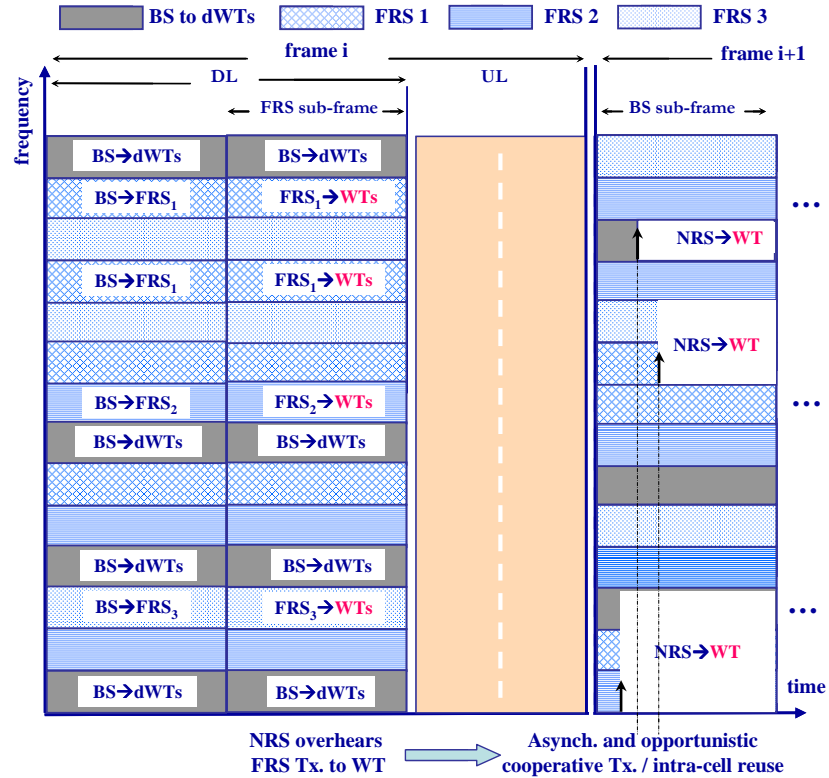

Fig. 2. Frame structure of the proposed schemes using FRSs and NRSs.

that the potential serving FRSs are stationary, while the NRS can be equipped with the computational power for its beam to follow a mobile WT. That is because the NRS does not handle upper layer issues such as applications and human interfaces. Fixed power allocation for BSs and relays is assumed. The transmit power per subchannel of the BS is greater than that of an FRS and much greater than that of an NRS. The choice of low transmit power for NRS is informed by its proximity to the WT so that NRSs do not unnecessarily cause interference to other links. Continuous backlog is assumed at the BS buffers but not at the FRSs. In general, the basic operation of the schemes can be described as follows.

In the BS sub-frame, the BS transmits to the direct WTs (dWTs) and the FRSs with connected WTs while any NRS can access one or more subchannels at a random instant, immediately after listening to the activity on all subchannels, and transmit to a particular WT during the remaining time of the same sub-frame. The NRS's opportunistic medium access results in intra-cell (or intra-region) reuse if an acquired subchannel is allocated to a BS-FRS feeder link or a BSdWT access link. In the FRS sub-frame, the BS continues to communicate with the dWTs on the same set of subchannels assigned to them in the previous sub-frame; all channels are invariant over the DL frame. FRSs transmit to their connected WTs while the NRSs overhear and always decode the transmissions to their respective WTs.

WTs can receive from multiple nodes simultaneously on orthogonal subchannels. This happens if a WT is connected directly to the BS while receiving from its NRS. Note that due to the half-duplex operation, an NRS may not overhear and transmit concurrently. For the sake of illustration and emphasizing the concept, we consider a protocol where NRS cooperates with only FRSs ${ }^{1}$. Therefore, the NRS transmits during the next BS sub-frame, after receiving from the FRS during the FRS sub-frame of the past DL frame. Such protocol

\footnotetext{
${ }^{1}$ Based on the dynamic routing, different protocols can be devised by imposing constraints on either the routing options or the NRS assistance to facilitate its cooperation with the BS's and/or the FRS's transmissions.
}

is different from ARQ through the serving FRS for the following reasons: 1- Latency is significantly less since the NRS reacts after one UL frame whereas the serving FRS can only retransmit after a whole TDD frame duration elapses. 2- The FRS would have to utilize a portion of the system's premium resources to retransmit whereas the NRS cooperates through intra-cell reuse. 3- The protocol may exploit the spatial diversity offered by the FRS-NRS link which enjoys a better link budget through the NRS directional antenna gain.

We now address the feedback required to realize the proposed schemes. The channel state information (CSI) in the form of per-subchannel achievable rate is available at the transmitting nodes as follows; for a BS-dWT or an FRS-WT link the terminal needs to feedback its CSI to the serving node; this is a vector of length $N$. Whereas for an NRS-WT link, no feedback is required from the WT to its NRS. On a BS-FRS link, the FRS reports the quality of this feeder as well as the second hops, if any, based on the operating scheme:

- Semi-centralized: A matrix of dynamic size $N \times K_{m}$ reporting the CSI of $K_{m}$ connected WTs.

- Distributed: Two $N \times 1$ vectors representing the processed output of the FRS, as explained in Fig. 3.

\section{The Dynamic Routing or Relay Selection}

We referred to this routing strategy as WT-based routing or link selection. Each WT can be served directly by the BS or through an FRS. The challenge is how the WT compares the access links while the quality of each varies over $N$ subchannels. A key fact is that a WT will be scheduled on only a subset of the $N$ subchannels on the selected link; such subset potentially encompasses the link's best subchannels rather than the poor ones. As such, during DL frame $i$, the $k$-th WT assesses the quality of the subchannels on the $M+1$ access links, computes a statistical metric based only on the best percentile of subchannels on each link (e.g., the mean value), and then selects the link with the largest metric. The feedback is sent to only the selected node during the uplink frame $i$. Hence, the routing decision is known to the concerned node and implicitly to all other nodes with substantial savings in feedback overhead. These steps are the same for both the distributed and semi-centralized schemes. There is a tremendous advantage in this routing strategy compared to the conventional approach where the subchannels on each potential serving link are reported. Note that further savings are achieved by replacing the continuous achievable rates by indexes of the discrete AM modes when AM lookup tables are used.

We define $\bar{R}_{m \rightarrow k}^{(i)}$ as the average achievable rate of the selected percentile on the user's access link from node $m$ during the DL frame $i$. The $k$-th WT's route selection is then based on the following criterion:

$$
\mathcal{K}_{m^{*}}^{(i+1)} \leftarrow \mathcal{K}_{m^{*}}^{(i+1)} \cup\{k\}, \quad m^{*}=\arg \max _{m}\left\{\bar{R}_{m \rightarrow k}^{(i)}\right\}, \forall m,
$$

where $\mathcal{K}_{m}^{(i+1)}$ is the set of WTs connected to node $m$ during DL frame $i+1 .^{2}$

\footnotetext{
${ }^{2}$ The frequency of executing this strategy can be generally relaxed in time so that the routing decision is changed after averaging the metric over a window of several frames.
} 


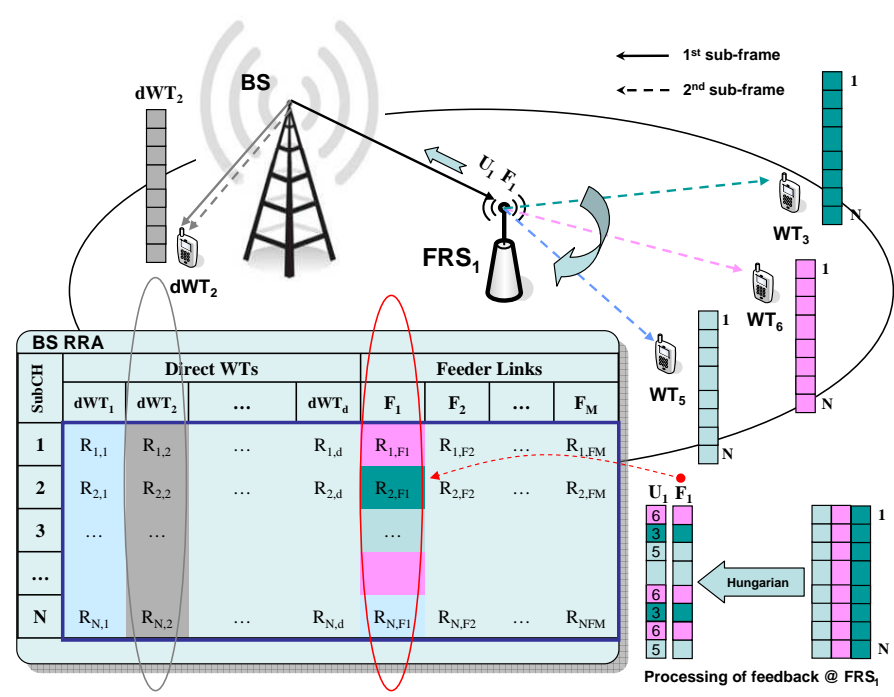

Fig. 3. Illustration of the operation of the distributed RRA scheme and the achievable rates matrix at the BS. In the semi-centralized scheme, the column of feeder link $m$ is replaced by the $N \times K_{m}$ rate matrix without processing.

\section{Radio Resource Allocation at the BS}

The BS's radio resource allocation (RRA) algorithm for both schemes operates in a greedy manner to maximize the overall system throughput under the users' QoS constraints, including the cell-edge users. The QoS requirements are represented by the user's minimum rate $R_{\text {min }}$ and the target BER $P_{e}$. Based on the earlier assumptions and system model, the BS can allocate the resources dynamically among all the dWTs and the FRSs as a two-dimensional assignment problem. Therefore, the Hungarian algorithm [8] is used to provide a low-complexity iterative solution in which each iteration is solved optimally and incurs a polynomial complexity of $\mathcal{O}\left(\left(\max \left\{N_{u}, K_{0}+M_{a}\right\}\right)^{3}\right)$ in the distributed scheme, and $\mathcal{O}\left(\left(\max \left\{N_{u}, K\right\}\right)^{3}\right)$ in the semi-centralized, where $N_{u}$ and $M_{a}$ are respectively the number of unassigned subchannels and the number of feeder links with connected users. The matrix passed to the Hungarian algorithm is constructed using the achievable rates fedback by the FRSs and the dWTs as shown in Fig. 3. After each iteration, the minimum rate requirement of each column is compared to its assigned sum rate. When the rate requirement is satisfied for a column, it is excluded from the subsequent iterations. As such, the assignments are not necessarily one-to-one. Since the dWTs, unlike the relayed WTs, remain connected for the whole DL frame, the minimum rate requirement of a relayed WT is seen as double that of a dWT, given the equal sub-frames. If all columns are rate satisfied before the subchannels are exhausted, the operator has the flexibility to assign the remaining subchannels among the dWTs and/or the feeder links that have the highest achievable rates. To put more emphasis on the impact of NRSs, given the FRS-NRS cooperation protocol we adopted, the remaining subchannels are allocated in a greedy manner among the feeder links only, and thus among the users relayed through the FRSs.

\section{A. Distributed Scheme}

During the UL frame, each FRS combines the feedback of its $K_{m}$ connected users into a single vector through a different implementation of the Hungarian algorithm regardless of the minimum rate constraints. As shown in Fig. 3, this vector is evenly shared by the actual rates and therefore can be considered a wish-list that provides unbiased representation of the second hops altogether; this vector could be influenced, under different processing, by the worst or the best relayed user's link. In a related work [5], such processing is performed using the average or median, per subchannel, of the SINRs across all the connected users. As such, the reported values do not reflect on any user's actual subchannel quality. Moreover, an overlooked problem arises from the BS's perspective: How many data bits should the BS send to each relayed user? We provide a neat solution to such problem; the indexed usersubchannel information is conveyed in two $N \times 1$ vectors to the BS which performs the optimization and then loads the estimated information bits for each relayed WT based on the rates selected from the WT's entries in the combined column.

We also note that when the feeder link appears as a single processed column in the BS RRA matrix, the feeder will have a virtual minimum rate requirement that is equal to double the sum of its connected users' rate requirements.

The RRA at the FRSs: Due to the combined feedback of relayed users, the BS RRA does not guarantee that the rate requirement of each user will be met. Therefore, the FRS starts a separate reallocation process on the set of subchannels that has been assigned to its feeder link. The FRS performs a lowcomplexity optimization process similar to the BS's but on an $N_{m} \times K_{m}$ rate matrix. The rate constraints are checked after each assignment. Remaining subchannels after satisfying the constraints are assigned to the best users.

\section{B. Semi-centralized Scheme}

The main difference here is that the BS performs the RRA for the relayed WTs and the dWTs without involving the the FRSs. However, as explained in Section II, the scheme still has limited-feedback and routing is carried out by the WTs. The achievable rates of the relayed WTs at $\mathrm{FRS}_{m}$ are arranged in an $N \times K_{m}$ matrix and conveyed to the BS during the UL frame to form an $N \times K$ matrix.

\section{NOMAdic RElay OpERATIONS}

Figure 4 demonstrates the operations of the NRS in resource acquisition and cooperation. During the FRS sub-frame, the FRS transmits to its connected WTs on the specified subchannels while the BS continues to transmit to the dWTs. An NRS dedicated to a particular WT always overhears and decodes the transmissions from the serving FRS. In the next BS subframe, the BS transmits to some dWTs and FRSs while the NRS can access one or more subchannels to forward the data it has reliably decoded to the WT spontaneously or based on the WT's request upon reception of erroneous data segments. Generally, some combining techniques (selection or maximal ratio combining) can be employed at the WT thereafter.

\section{A. Subchannels Acquisition and Selective Retransmission}

The important issue here is how the NRS autonomously chooses the subchannel(s) it needs to communicate with the WT. The NRS chooses a random instant to listen to the whole bandwidth and estimates, without detection, the amount of received power on each subchannel (Fig. 4). Based on the proximity of the NRS to the WT, the total power received at the NRS on a subchannel during this short listening period can be a good approximation of the interference level that could be 

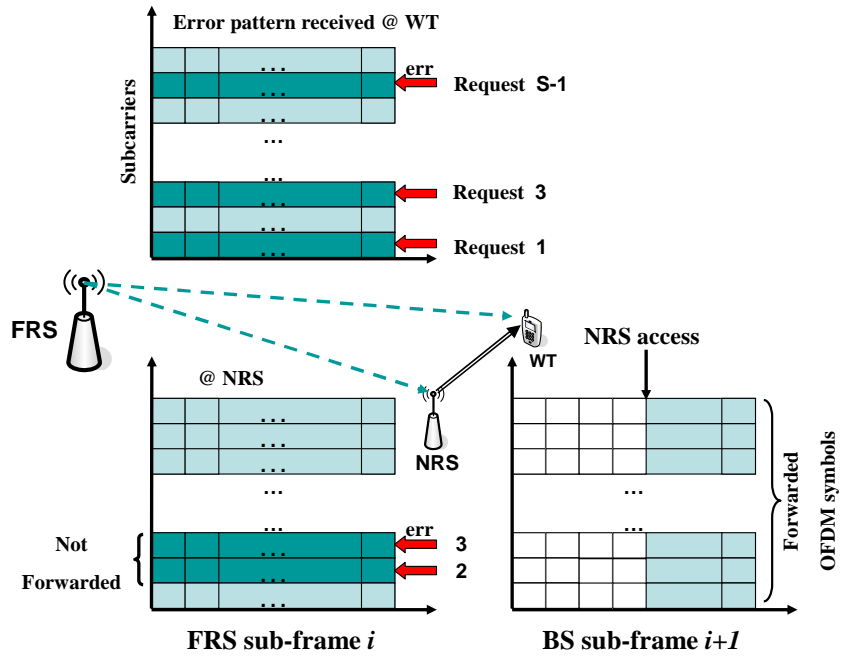

Fig. 4. Illustration of the cooperation of the serving FRS and the dedicated NRS to assist the WT. $S$ is the number of OFDM subcarriers per subchannel.

observed by the WT if the NRS uses that subchannel for the remaining sub-frame duration. The NRS will examine all the $N$ subchannels except those assigned to the WT (if connected directly to the BS in that frame). Due to the asynchronous access, the probability of having more than one NRS listening at the same instant, and furthermore, interfering significantly with each other's transmission, is negligible. The NRS sorts the observed subchannels based on the estimated total received power and acquires the least-power subchannel(s). The number of chosen subchannels can be determined if we consider that the NRS attempts to minimize latency by forwarding all the data segments it received properly, and the WT requires, during the remaining BS sub-frame duration and using the same AM modes (see Fig. 2). The key idea behind asynchronous access is to allow the NRS to sense the activity of other NRSs that are close in distance, or more precisely, with high interfering-link gains; thus minimizing the likelihood of choosing the same subchannels immediately acquired by such NRSs. A similar idea to our asynchronous listening and updating has been employed for ad-hoc networks in [7] where they rely strongly on channel reciprocity so that a cluster head can estimate the interference it will cause to others when it uses a particular band. However, this condition is not necessary in our schemes since the NRS only listens to estimate the interference that its WT will experience on a subchannel if the NRS uses it to serve the WT. It is worth mentioning as well that the medium access aspect of the LARA protocol is different from the carrier-sense multiple access (CSMA) technique adopted in the 802.11 standards: LARA is not contention-based and operates in an OFDMA-based cellular system where excessive $\mathrm{CCI}$ is avoided through acquiring the carriers with the least activity observed. A subchannel could be acquired by several NRSs without a back-off mechanism, given the proximity of the NRS to its WT and the low NRS transmit power.

\section{B. NRS-FRS Cooperation}

While the 'data segment' on which the NRS selective transmission operates may refer to any data unit such as a packet or a subchannel, we here consider, without loss of generality, a subcarrier as the data segment as shown in Fig. 4.
TABLE I

SYSTEM PARAMETERS: ADOPTED FROM THE WIMAX FORUM.

\begin{tabular}{|c|c|}
\hline \hline Parameter & Value \\
\hline User min. close-in distance to BS & $35 \mathrm{~m}$ \\
\hline BS Tx. antenna gain & $15 \mathrm{~dB}$ \\
\hline FRS Tx. antenna gain & $10 \mathrm{~dB}$ \\
\hline WT Rx. antenna gain & $0 \mathrm{~dB}$ \\
\hline Shadowing st. dev. on user and interference links (NLOS) & $8.9 \mathrm{~dB}$ \\
\hline Shadowing st. dev. on FRS-NRS links (NLOS) & $7 \mathrm{~dB}$ \\
\hline Shadowing st. dev. on NRS-WT links (LOS) & $1.5 \mathrm{~dB}$ \\
\hline Carrier frequency & $2.5 \mathrm{GHz}$ \\
\hline Total bandwidth & $20 \mathrm{MHz}$ \\
\hline User mobility & $10 \mathrm{Km} / \mathrm{hr}$ \\
\hline Channel sampling time = TDD frame length & $5 \mathrm{msec}$ \\
\hline Downlink : Uplink ratio & $2: 1$ \\
\hline DL Tx. time in OFDM data symbols & $24 \mathrm{symbols}$ \\
\hline OFDM subcarrier bandwidth & $10.9375 \mathrm{KHz}$ \\
\hline OFDM symbol duration & $102.86 \mu \mathrm{sec}$ \\
\hline Subchannel width & $18 \mathrm{subcarriers}$ \\
\hline Noise power density at Rx. nodes & $-174 \mathrm{dBm} / \mathrm{Hz}$ \\
\hline BS total Tx. power & $46 \mathrm{dBm}$ \\
\hline RS total Tx. power & $37 \mathrm{dBm}$ \\
\hline NRS total Tx. power & $19 \mathrm{dBm}$ \\
\hline \hline
\end{tabular}

By selective 'retransmission', the NRS only requires a fraction of the resource used by the FRS to forward its data; this is particularly useful since the NRS only accesses the channel over a fraction of the BS sub-frame.

\section{Simulation Results}

The same QoS requirements (target minimum rate and maximum BER) are desired for all WTs. The minimum rate requirement is set to $250 \mathrm{Kbps}$ and the target BER is $10^{-3}$. The $10^{t h}$ percentile is used in the WT-based routing. The simulated cellular network consists of 19 hexagonal cells enhanced with 3 or 6 FRSs. These relays are placed at a distance of 0.65 of the cell radius from the BS and with a uniform angular spacing. The distance between two adjacent BSs is $2 \mathrm{Km}$. Users are uniformly distributed within the cell area. It is assumed that the NRS is placed randomly within a distance of at most 20 $\mathrm{m}$ from the WT. Time-frequency correlated small-scale fading is assumed using WINNER C2 channel models. Independent lognormal shadowing is considered for different links in the network. As for the FRS-WT link and the corresponding FRSNRS link, the same shadowing realization is applied to both links as different shadowing spatial correlation models result in an almost unity correlation coefficient. The path-loss model for the links as a function of the distance (in meters) is given as $P L=38.4+35 \log _{10}(d) \mathrm{dB}$. Each FRS has an omnidirectional transmit antenna to communicate with the WTs as well as a highly directive receive antenna aiming at the BS. A list of channel and system parameters used for the simulations are given in Table I. The time-average and instantaneous user throughput: The CDFs of the time-averaged throughput in Fig. 5 show the throughput gains due to the proposed NRS cooperation to assist the relayed WTs as well as the cell-edge performance under the proposed schemes. According to the LTE evaluation methodology, the $5^{t h}$ percentile throughput corresponds to the cell-edge. It can be observed that given the same $K$ and $M$, the semi-centralized scheme outperforms the distributed in general and at the cell-edges in particular. This is due to the fact that the BS directly optimizes the RRA of the relayed users, who likely include the cell-edge 


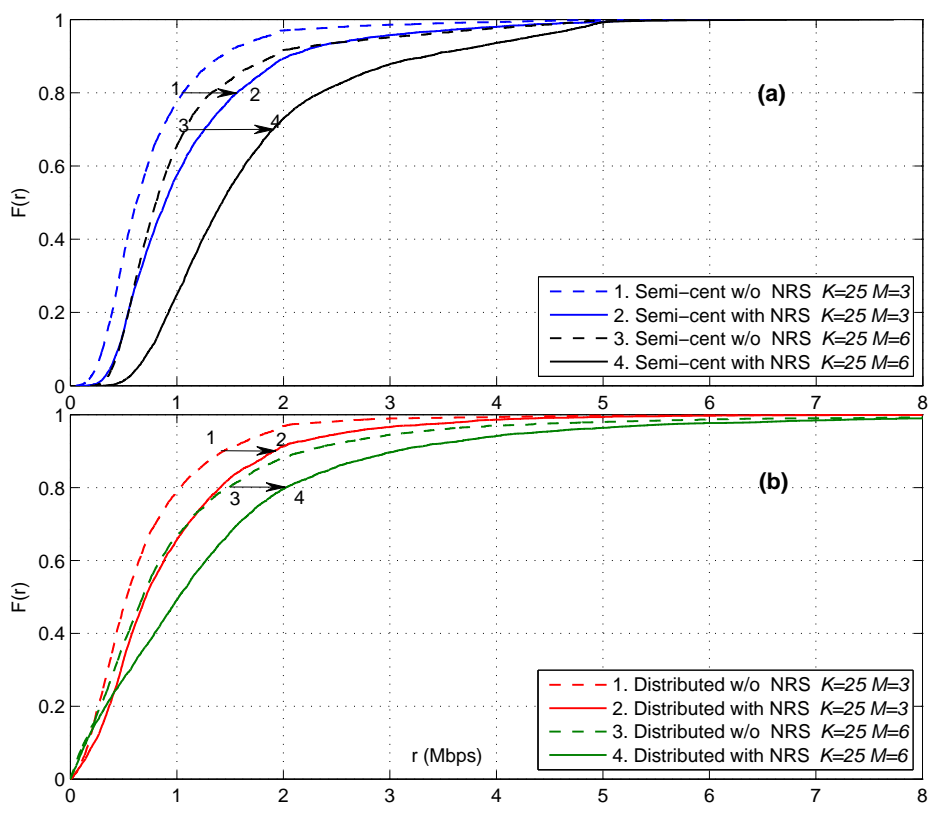

Fig. 5. CDF of the time-average user throughput for the distributed and semi-centralized schemes, using 3 or 6 FRSs, with and without NRSs, $K=$ $K_{\text {nom }}=25$.

TABLE II

OUTAGE PROBABILITY BASED ON USERS' INSTANTANEOUS THROUGHPUT RATES, $R_{\min }=250 \mathrm{KBPS}$.

\begin{tabular}{|c|cc|cc|}
\hline \hline & \multicolumn{2}{|c|}{ Semi-centralized } & \multicolumn{2}{c|}{ Distributed } \\
\hline Parameters & With NRS & w/o NRS & With NRS & w/o NRS \\
\hline$K=15 M=6$ & 0.0155 & 0.0280 & 0.2135 & 0.2686 \\
\hline$K=25 M=6$ & 0.0398 & 0.1416 & 0.2669 & 0.3315 \\
\hline
\end{tabular}

users due to the WT-based routing, using their individual feedback information rather than allocating the resources to the serving FRSs based on combined feedback and QoS requirements. Given the rate matrix composition, the semicentralized scheme better exploits the multi-user and spatial diversities than the distributed.

The performance gain due to the NRS assistance is clearly evident in both schemes. However, due to the considered uniform distribution of FRSs and WTs, and the WT-based routing strategy which is common in both schemes, the number of relayed users increases as the number of FRSs increases. Since the NRS cooperation we considered is limited to the relayed users, the performance gain due to the employment of NRSs is amplified as the number of FRSs increases. The same relative performances are realized through the outage probabilities in Table VI based on the user's instantaneous throughput rate $R_{k}$ achieved in each DL frame, as compared to the minimum required rate, i.e., $\mathcal{P}\left\{R_{k}<R_{\text {min }}\right\}$.

The network reuse factor represents the number of times a subchannel is utilized concurrently (same BS sub-frame) all over the cellular network comprising $N_{R}$ 'regions' or cells, and normalized by $N_{R}$. Statistics are collected from all subchannels and $N_{R}=19$ cells. Note that in the schemes without NRS, a unity network reuse factor should be always realized. Observing a histogram of such statistics, network reuse realizations of 1.5 and up to 2 are obtainable; since the counts are discrete integers, this means that a subchannel might be opportunistically reused twice or more in a cell as compared to the static reuse patterns which limit the degrees of freedom
TABLE III

MEAN NETWORK REUSE FACTOR FOR THE PROPOSED DISTRIBUTED AND SEMI-CENTRALIZED SCHEMES.

\begin{tabular}{|c|c|c|c|}
\hline \hline$K$ & $M$ & Distributed & Semi-centralized \\
\hline 15 & 3 & 1.2319 & 1.3156 \\
\hline 15 & 6 & 1.2351 & 1.3655 \\
\hline 25 & 3 & 1.2448 & 1.3387 \\
\hline 25 & 6 & 1.2745 & 1.4282 \\
\hline
\end{tabular}

in the system. Due to space limitations we only show the mean values of these realizations in Table III which also attests to this fact. Increasing the number of WTs in both schemes, and thus the number of assisting NRSs, results in the occurrence of higher reuse factors. In addition, the dynamic routing strategy in a network with denser FRS deployment results in more relayed users through the FRSs to which NRS cooperation is tied; this means more subchannel reuse. In general, the semi-centralized scheme provides higher average reuse factor than the distributed. Note that the semi-centralized scheme can satisfy the rate requirements using less subchannels and therefore more remaining subchannels are assigned to the relayed users who invoke the NRS cooperation more often.

\section{CONCLUSIONS}

This paper presents radio resource management schemes and techniques to facilitate the operation of the envisioned nomadic relay-augmented fixed relay networks using OFDMA technology. The RRM schemes are classified as being distributed or semi-centralized, with respect to the role of the fixed relay stations. A nomadic relay, an add-on to a relaybased network, operates autonomously (in both schemes) to acquire its own radio resources without depending on a central entity for allocation. A number of techniques such as, user-based link selection, opportunistic intra-cell reuse, and nomadic-fixed relay cooperation, have also been incorporated into the study. Through comprehensive performance evaluation of the proposed systems, schemes, and techniques, we have been able to establish the proof of concept of nomadic relayaugmented fixed relay networks.

\section{REFERENCES}

[1] IEEE P802.16j/D1, "Draft IEEE Standard for Local and metropolitan area networks Part 16: Air interface for fixed and mobile broadband wireless access systems: multihop relay specification," pp. 1002-1007, August 2007.

[2] IEEE 802.16, "Mobile multihop relay study group recommendations for the scope and purpose of the mobile multihop relay task group," 11 November 2005, http:www.ieee802.org/16/sg/mmr/contrib/C80216mmr05_032.pdf.

[3] D. Soldani and S. Dixit, "Wireless relays for broadband access," IEEE Communications Magazine, 46(3), pp. 58-66, March 2008.

[4] X. Qiu and K. Chawla, "On the performance of adaptive modulation in cellular systems," IEEE Transactions on Communications, 47(6), pp. 884-895, June 1999.

[5] M. K. Kim and H. S. Lee, "Radio resource management for a two-hop OFDMA relay system in downlink," IEEE Symposium on Computers and Communications (ISCC), pp. 25- 31, July 2007.

[6] J. Lee, S. Park, H. Wang, and D. Hong, "QoS-gurarantee transmission scheme selection for OFDMA multi-hop cellular networks," IEEE International Conference on Communications (ICC), pp. 4587-4591, June 2007.

[7] B. Babadi and V. Tarokh, "A distributed asynchronous algorithm for spectrum sharing in wireless ad hoc networks," Annual Conference on Information Sciences and Systems (CISS), pp. 831-835, March 2008.

[8] J. Munkers, "Algorithms for the assignment and transportation problems," Journal of the Society for Industrial and Applied Mathematics, pp. $3238,1957$. 